\title{
VALIDEZ DEL USO DE LA FOTOGRAFÍA COMPUTARIZADA EN LA EVALUACIÓN DE LA AM- PLITUD DEL MOVIMIENTO DE LA RODILLA UTILIZANDO LA GONIOMETRÍA DE LA IMAGEN RADIOLÓGICA COMO PADRÓN ORO
}

\author{
VALIDITY OF THE USE OF COMPUTERIZED PHOTOGRAPHY FOR THE EVALUATION OF THE \\ KNEE MOTION RANGE USING THE GONIOMETRY OF THE X-RAY IMAGE AS A GOLD STANDART
}

Coelho, Renato Ramos ${ }^{\text {; }}$ Batista, Luiz Alberto ${ }^{2}$; Cunha, Gerson Gomes ${ }^{3} \&$ Dantas, Estélio Henrique Martin ${ }^{4}$.

1 Laboratório de Biociência da Motricidade Humana (LABIMH), Laboratório de Métodos Computacionais em Engenharia (LAMCE/COPPE/ UFRJ). - Rio de Janeiro, Brasil

2 Universidade do Estado do Rio de Janeiro (UERJ) - Rio de Janeiro, Brasil

3 Laboratório de Métodos Computacionais em Engenharia (LAMCE/COPPE/UFRJ) - Rio de Janeiro, Brasil

4 Programa de Pós-graduação Stricto Sensu em Enfermagem e Biociências (PPgEnfBio) da Universidade Federal do Estado do Rio de Janeiro (UNIRIO) - Rio de Janeiro, Brasil

COELHO R.R.; BATISTA L.A.; CUNHA G.G. \& DANTAS E.H.M. Validez del uso de la fotografía computarizada en la evaluación de la amplitud del movimiento de la rodilla utilizando la goniometría de la imagen radiológica como padrón oro.Mot. Hum. 13 (1): 45 -50; 2012.

\begin{abstract}
RESUMEN
En la investigación se hace una observación de la validez de la utilización de la fotografía computarizada (FC) para evaluar la amplitud del movimiento (ADM) de la rodilla. Se hice la verificación de la reproducción de mediciones obtenidas por diferentes examinadores y una comparación de los resultados obtenidos por el uso de la FC con aquellos obtenidos por el padrón oro: la goniometría de la imagen radiológica (GIR). Doce personas del curso de Fisioterapia fueron utilizadas, divididas en dos grupos: 1. Un profesor y tres estudiantes, actuando como examinadores $(25.75+$ 2.99 años, con experiencia mínima en anatomía de superficie de 2.5 años), responsable por la obtención de datos de ADM de la rodilla; 2 . Ocho personas actuaron como evaluadas (edad media de $30.13+10.13$ años, sin histórico de patologías en la rodilla izquierda), los cuales tuvieran su rodilla izquierda analizada. Cada evaluado fue analizado por 2 examinadores en un total de 32 mediciones. Para la evaluación vía FC se han utilizado los siguientes puntos anatómicos: trocánter mayor, condillo femoral lateral y maléalo lateral. Para la GIR, se ha utilizado una imagen radiológica en perfil de la misma rodilla. El coeficiente de correlación intraclase ha indicado una objetividad mínima de 0.997 y la prueba de Pearson ha indicado una correlación de 0.987 entre las mediciones obtenidas por la FC y por la GIR. Los resultados obtenidos han permitido verificar que la FC posee una gran validez para la evaluación de la ADM de la rodilla.
\end{abstract}

Palabras clave: validez, fotografía computarizada, amplitud del movimiento, rodilla.

This study verifies the validity of the use of compu

terized photography $(C P)$ to evaluate the knee range of motion (ROM). In doing so, it was verified the objectivity of collected data by different appraisers and the correlation between the results obtained from $C P$ and from the goniometry of the X-ray image (GXI). A group of 12 people was used (students and teachers) divided into 2 groups. One group acted as appraiser $(25.75+2.99$ years age $)$, with a minimum experience of 2.5 years in superficial anatomy, evaluating the knee ROM $(n=4)$; the other group, formed by people without any history of injury in the left knee $(30.13+10.13$ years of age, $n=8)$, had had their left knee evaluated by two appraisers from the first group, in a total of 16 measurements. For the evaluation made by the $C P$, it was used three anatomic references: trochanter major, lateral femoral condyle and lateral maleollus of the left lower limb. To the GXI, it was used a lateral image of the left knee. The intraclass correlation coefficient indicates a minimum objectivity of 0.997 and the Pearson's Test indicate a correlation of 0.987 between the data obtained by the CP and that obtained by the GXI. These results are an indication that the CP has a great validity for the evaluation the knee ROM.

Key words: validity, computerized photography, range of movement (ROM), knee. 
COELHO R.R.; BATISTA L.A.; CUNHA G.G. \& DANTAS E.H.M. Validez del uso de la fotografía computarizada en la evaluación de la amplitud del movimiento de la rodilla utilizando la goniometría de la imagen radiológica como padrón oro..Mot. Hum. 13 (1): 45-50; 2012.

\section{INTRODUCCIÓN}

La morfología del miembro inferior y su biomecánica es un importante componente de la aptitud física, está relacionada a la salud y a las condiciones atléticas (1). La evaluación de eso está condicionada a algunos hechos tales como: 1. Las dificultad de si evaluar el miembro en condiciones dinámicas; 2. La falta de medidas padrón validadas; 3 . La dificultad en evaluar una articulación sin involucrar otras que la circundan (2). Para la manipulación de estos factores y su cotejo, han sido desarrollados diferentes métodos de evaluación de la biomecánica.

Las más recomendables son las pruebas que expresan resultados en grados de amplitud del movimiento (ADM) de la articulación (3).

El padrón oro para esa evaluación angular es la goniometría realizada con imagen radiológica (GIR) de la articulación (4). Debido a los costes y riesgos inherentes a la radiología, ese método trae problemas cuando se evalúan criaturas humanas (5). En este contexto, diferentes aparatos han sido utilizados reemplazando este padrón oro, tales como: goniómetro (3), electrogoniómetro (6) y fotografía computarizada (FC). Este último tiene su utilización restricta y dependiente de programas validados y de controles de evaluación que posean buena validez y confiabilidad (4).

El término validez remite a la credibilidad y a la precisión de un estudio, estando presente solamente cuando los investigadores realmente observan lo que ellos se propusieron a estudiar (7). En este contexto, es fundamental el cotejo del dato obtenido por un método como aquel obtenido por el padrón oro de esa evaluación para que él sea validado (8). Un otro factor determinante para que un método tenga validez es una buena reproducción de los resultados obtenidos a través del mismo (9). La reproducción, por su vez, es un término que indica la consistencia de los resultados después de repetidas mediciones, hechas con un mismo voluntario y que puede ser dividida en dos tipos: 1. Confiabilidad, que indica la habilidad de un examinador en estar acorde con él mismo después de varias mediciones, utilizando un mismo método, en una misma persona. 2. Objetividad, la cual indica la consistencia de los resultados de una medición entre evaluadores diferentes, utilizando un mismo método, en una misma persona. Ese último visa eliminar la interferencia del evaluador en la obtención de datos en el cotejo de resultados de uno o de otro (10).

Computer Aided Design (CAD) es un grupo de software que usa una misma metrología. En estudios realizados con anterioridad, este tipo de software ha presentado una bue- na validez para la evaluación biomecánica de trabajo (4, 11). Todavía, faltan estudios que verifiquen la validez de su utilización en la evaluación de la ADM de la rodilla en la evaluación de la biomecánica entrenamiento deportivo.

El desarrollo del control para la evaluación utilizando la FC tiene que ver con la posición de la cámara fotográfica en relación al objeto a ser evaluado y cuales puntos serán utilizados como referencia (12). En trabajos desarrollados en humanos, los puntos de referencia preferenciales son las estructuras anatómicas superficiales que pueden ser localizadas a través del palpamiento, siendo que la palpación de prominencias de los huesos presenta una mayor confiabilidad que aquella hecha en los tejidos blandos (13).

Además de esto, es importante hacer aciertos en las distorsiones inherentes al proceso para la obtención de la fotografía (12). Para tal, es necesario que la cámara fotográfica utilizada en el proceso esté calibrada (14).

De ahí que la evaluación de la ADM por la FC es dependiente de la posición de la cámara fotográfica, de su calibración, de la posición de la persona a ser evaluada, de la habilidad del evaluador en localizar puntos de referencia por la palpación y de la utilización de software validado. De todo lo anterior, el objetivo de este estudio fue conferir validez para la utilización de la FC, analizada por software CAD, para la evaluación de la ADM de la rodilla en el plano sagital. Para esto, fue verificada la objetividad de tales medidas y la correlación entre aquellas obtenidas por la FC y las obtenidas por la GIR.

La presente validación posibilitará la utilización de esta técnica para la evaluación de la ADM de la rodilla.

\section{MATERIAL Y MÉTODOS}

Selección de una muestra por conveniencia de cuatro examinadores, tres académicos y uno profesor del curso de fisioterapia $(25.75+2.99$ años), todos con más de 2.5 años de experiencia en la localización, por palpamiento, de estructuras anatómicas.

También, por conveniencia, ha sido seleccionado un grupo a ser evaluado, compuesto por ocho estudiantes de fisioterapia $(30.13+10.13$ años $)$, sin histórico de patologías ortopédicas en los huesos del miembro inferior izquierdo (MII). Esa muestra fue escogida de manera a producir un grupo lo más heterogéneo posible a lo que se refiere al sexo, edad, peso, altura y indicador de masa corporal (IMC), como muestra la Tabla I. Estos cuidados han posibilitado un aumento de la validez externa del presente estudio (7). 
COELHO R.R.; BATISTA L.A.; CUNHA G.G. \& DANTAS E.H.M. Validez del uso de la fotografía computarizada en la evaluación de la amplitud del movimiento de la rodilla utilizando la goniometría de la imagen radiológica como padrón oro..Mot. Hum. 13 (1): 45-50; 2012.

\begin{tabular}{|c|c|c|c|c|c|}
\hline & $\mathrm{N}$ & Mínimo & Máximo & Media & SD \\
\hline Edad & 8 & 18.00 & 43.00 & 30.13 & 10.13 \\
\hline Peso & 8 & 47.50 & 89.00 & 66.81 & 12.85 \\
\hline Altura & 8 & 1.60 & 1.84 & 1.71 & 0.09 \\
\hline IMC & 8 & 18.56 & 26.29 & 22.74 & 2.65 \\
\hline
\end{tabular}

El presente estudio atendió a las normas éticas para la realización de investigaciones con seres humanos, conforme a todos los procedimientos de la declaración de Helsinki (15) y fue aprobado por el Comité de Ética en Investigación de la Universidad de Castelo Branco/ RJ, con el protocolo número 0029/2007.

Cada uno de los evaluados fue examinado por dos diferentes examinadores y, visando a verificar la objetividad del método propuesto, cada experto no tuvo acceso a los resultados obtenidos por los otros.

La metodología de la evaluación de la ADM de la rodilla fue desarrollada como sigue: 1. Posicionar el evaluado en supino; 2. Localización y identificación con un marcador de piel radiopaco del maléalo lateral, del condillo

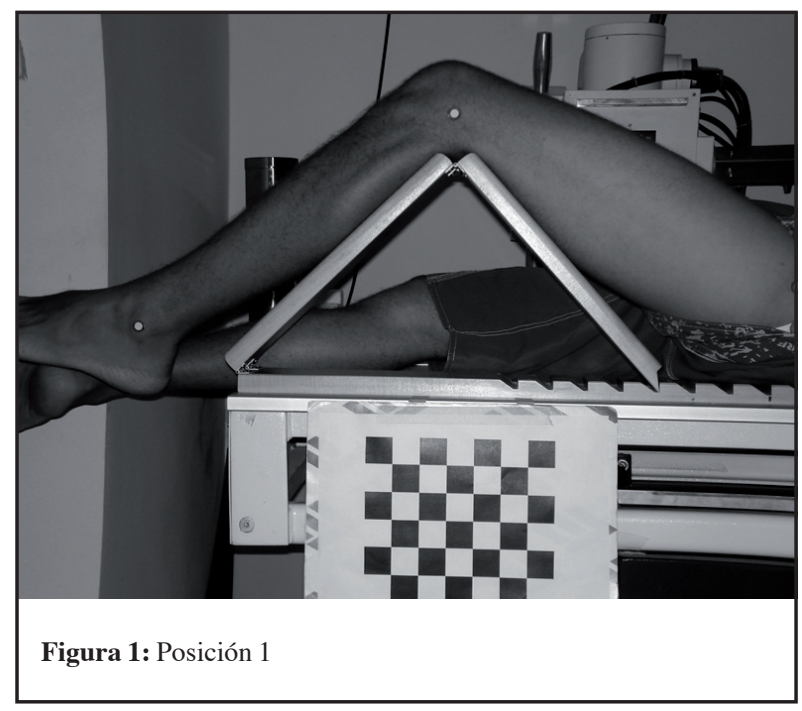

Para la GIR, el aparato de Rayos X fue posicionado en la dirección de la interlínea articular de la parte interna de la rodilla izquierda (16). Después se ha seguido el método descrito por Coelho (4): se ha hecho una recta correspondiente a la bisección de la diáfisis de la tibia y otra del fémur, medidas con una escala de 1:1000 (Trident, Brasil). El ángulo correspondiente a la flexión de la rodilla, for- lateral de la rodilla y del trocánter mayor en el MII del evaluado; 3. Posicionar el evaluado sobre una tabla de cuádriceps (ISP, Brasil); 4. Radiografiar el perfil de la rodilla izquierda, lo que fue hecho por un técnico habilitado; 5. Fotografiar la misma rodilla en la misma posición, lo que fue hecho por el examinador.

La tabla de cuádriceps fue utilizada para que el MII del evaluado se mantuviera en una misma posición durante la evaluación fotogramétrica y radiológica. El MII fue posicionado en dos ángulos diferentes: 1. Rodilla en mayor flexión (posición 1); 2. Rodilla más cercana de la extensión total (posición 2), como se puede observar en las Figuras 1 y 2. Cuatro voluntarios fueron evaluados en cada una de esas posiciones.

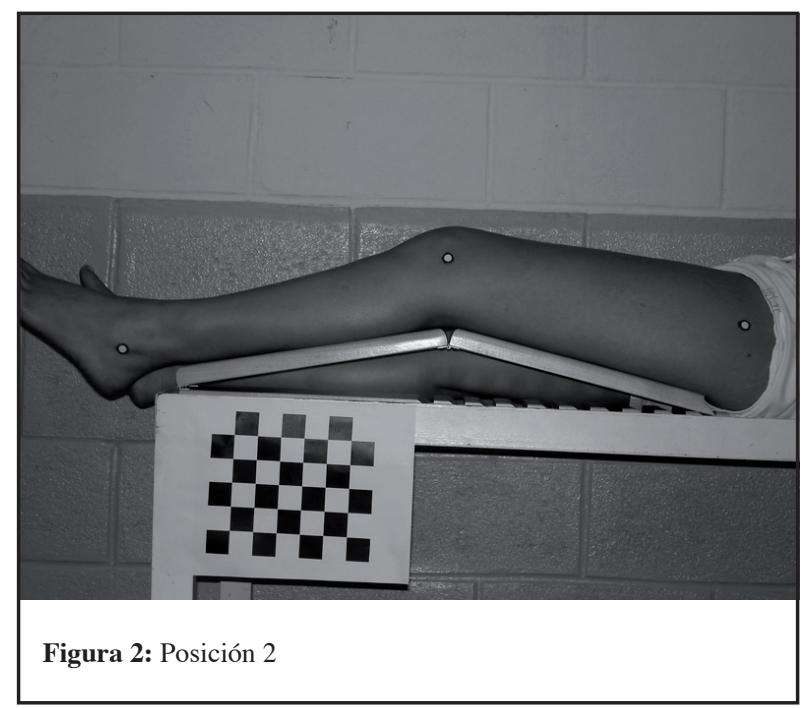

mado entre estas dos rectas, ha sido medido a través de la goniometría (Goniómetro Cardiomed, Brasil).

La metodología utilizada para la FC ha sido la cámara fotográfica (Sony, Nipón) posicionada como sigue: 1. Localizada a 2 metros de distancia de la rodilla; 2 . Perpendicular a la articulación de la rodilla del evaluado; 3 . Nive- 
COELHO R.R.; BATISTA L.A.; CUNHA G.G. \& DANTAS E.H.M. Validez del uso de la fotografía computarizada en la evaluación de la amplitud del movimiento de la rodilla utilizando la goniometría de la imagen radiológica como padrón oro..Mot. Hum. 13 (1): 45-50; 2012.

lada, paralelamente al suelo, sobre un trepé (Troni, Brasil); 4. Utilización de un encuadramiento que incluyera en el área de la fotografía el objeto evaluado y el cuadro para hacer la calibración de la cámara fotográfica (17), bajo el sistema GML Camera Calibration toolbox v. 04 (18).

En seguida, las fotografías digitales calibradas fueron analizadas por el CAD con la intención de verificar el ángulo en el cual la articulación de la rodilla se encontraba cuando en la tabla de cuádriceps. Estos ángulos fueron entonces analizados estadísticamente con el objetivo de determinar el valor de la correlación entre los datos obtenidos por cada uno de los métodos evaluativos. Para eso, fue utilizado el coeficiente de correlación intraclase tipo 2,3 (ICC) para verificar la objetividad de los valores obtenidos por cada uno de los examinadores. Así mismo, fue hecha la opción por la utilización de la prueba de Pearson para verificar la correlación $(\mathrm{R})$ entre los datos obtenidos por la GIR y por la FC. La significancia estadística adoptada presenta $\mathrm{p}<0,05$.

\section{RESULTADOS}

Los datos obtenidos por los examinadores para mediciones de ADM de la flexión de la rodilla, en la GIR, están descritos en la Tabla II, los obtenidos por los examinadores para las medidas de ADM de la flexión de la rodilla, en la FC, están descritos en la Tabla III.

\begin{tabular}{|lccccccc|}
\hline & N & Mínimo & Máximo & Media & SE & SD \\
\hline Posición 1 & 8 & 112 & 125 & 118.63 & 1.71 & 4.84 \\
Posición 2 & 8 & 156 & 164 & 159.13 & 0.99 & 2.80 &
\end{tabular}

Tabla II: Medidas de la ADM de la flexión de la rodilla en la GIR. Valores mínimos, máximos, media, error padrón (SE) y SD.

\begin{tabular}{|lcccccc|}
\hline & N & Mínimo & Máximo & Media & SE & SD \\
\hline Posición 1 & 8 & 109 & 123 & 116.38 & 1.52 & 4.31 \\
Posición 2 & 8 & 151 & 163 & 156.13 & 1.37 & 3.87 \\
\hline \\
\hline
\end{tabular}

Los datos relativos a la objetividad de los datos obtenidos, calculado a través del ICC, están descritos en la Tabla IV

\begin{tabular}{|c|c|c|c|c|c|c|c|c|c|}
\hline & \multirow{2}{*}{\multicolumn{2}{|c|}{ Examinador }} & \multirow[b]{2}{*}{$\mathrm{ICC}$} & \multicolumn{2}{|c|}{$\begin{array}{c}\text { Intervalo de } \\
\text { confianza de } 95 \%\end{array}$} & \multicolumn{2}{|c|}{$\begin{array}{c}\text { Prueba } F \text { con } \\
\text { valor verdadero } 0\end{array}$} & \multirow[b]{2}{*}{ df2 } & \multirow[b]{2}{*}{$\mathrm{P}$} \\
\hline & & & & $\begin{array}{l}\text { Límite } \\
\text { inferior }\end{array}$ & $\begin{array}{l}\text { Límite } \\
\text { superior }\end{array}$ & Valor & df1 & & \\
\hline \multirow[t]{2}{*}{ GIR } & $1 \times 2$ & Media & 0.99 & 0.97 & 1.000 & 467.98 & 3 & 3 & 0.00 \\
\hline & $3 \times 4$ & Media & 0.99 & 0.96 & 1.000 & 394.55 & 3 & 3 & 0.00 \\
\hline \multirow[t]{2}{*}{ FC } & $1 \times 2$ & Media & 0.99 & 0.97 & 1.000 & 490.57 & 3 & 3 & 0.00 \\
\hline & $3 \times 4$ & Media & 0.99 & 0.96 & 1.000 & 352.04 & 3 & 3 & 0.00 \\
\hline
\end{tabular}


COELHO R.R.; BATISTA L.A.; CUNHA G.G. \& DANTAS E.H.M. Validez del uso de la fotografía computarizada en la evaluación de la amplitud del movimiento de la rodilla utilizando la goniometría de la imagen radiológica como padrón oro..Mot. Hum. 13 (1): 45-50; 2012.

La prueba de Pearson ha indicado la existencia de $\mathrm{R}=$ 0,987 entre las medidas obtenidas por la GIR y por la FC para un valor de $\mathrm{P}<0.05$. De este valor de correlación obtenido, fue calculado el valor de R2 con un resultado de 0.974 para un valor de $p<0.05$. Ese valor indica que los valores obtenidos cuando de la utilización de la FC son iguales a 97,4\% de los valores obtenidos por GIR.

\section{DISCUSIÓN}

Los resultados han indicado que la FC es un método válido para la obtención de datos relativos a la ADM de la rodilla en el plano sagital. Esta afirmación está basada en los buenos resultados obtenidos para la concordancia de los resultados de diferentes examinadores (objetividad mínima de 0.997) lo que está acorde con el facto que la objetividad es determinante para que un método tenga validez (9). Otro factor que abogó a favor de esta validez ha sido el valor de R (0.987), considerado como muy fuerte, y R2 (0.974), existentes entre las mediciones obtenidas por la FC y las obtenidas por la GIR (4).

Los valores de la objetividad obtenidos por la FC sugieren que ella es más eficiente para la evaluación de la ADM de la rodilla que métodos tradicionalmente utilizados en otros estudios. El goniómetro, aunque posea una buena confiabilidad prueba/contra-prueba (0.90 a 0.98 ), presenta heterogeneidad en su objetividad (variación de 0.25 a 0.91 ) lo que disminuye su aplicabilidad clínica y terapéutica (19). El electrogoniómetro posee una grande precisión para las mediciones de ADM, pero su objetividad es considerada como más pequeña que la aceptable debido a la dependencia que las mediciones electrogionométricas tienen de los sensores necesarios a su utilización (6).

Las mediciones por la FC también son dependientes de los sensores posicionados sobre estructuras anatómicas, estando, por lo tanto, sujetas al mismo problema identificado para la electrogoniometría. Estudios verificaran limitación para la evaluación de la rodilla debido a la utilización de la palpación para la localización de las estructuras de referencia $(4,19)$. Autores creen que este método es empírico y muy sujeto a los problemas de aferición debido a la heterogeneidad de su objetividad (20). En contra de esta afirmación nuestros resultados indicaron que el posicionamiento de los sensores utilizados, a través de la palpación, no trajo problemas a la validez de las mediciones obtenidas en los evaluados a través de la FC. Otro factor importante, y que no fue evaluado en este trabajo, fue el desplazamiento de la piel y de la marcación durante el movimiento (21). Este factor puede contribuir para que los resultados presenten un error de origen en sus mediciones, principalmente a si evaluar la ADM máxima, aunque no parece tener influencia en la validez del protocolo propuesto en los ángulos utilizados en este trabajo.

La buena validez da FC observada en el presente estudio también pudo ser encontrada en otros trabajos, los cuales, utilizaran otra versión del CAD, para evaluar ergonomía y biomecánica (4). Resultados semejantes y que también están acordes con los nuestros, fueron obtenidos para la utilización del mismo software para cotejar, en chapas radiológicas, los ángulos formados entre las vértebras de la columna lumbar (22).

\section{CONCLUSIÓN}

Los datos obtenidos, asociados al análisis estadístico utilizado, han permitido inferir que la FC analizada por el CAD es un método que presenta validez para la evaluación de la ADM intermediaria de la articulación de la rodilla en el plano sagital. Esto ha podido ser verificado a través del análisis de la objetividad de las medidas bien como por la comparación de este método con la GIR, considerada un padrón oro para este tipo de evaluación.

Como sugerencia a nuevos estudios en el área, se puede elaborar uno en el cual la rodilla no sea posicionada estáticamente como hecho en el presente trabajo, con la rodilla sobre la tabla de cuádriceps. Se puede también observar la precisión de esta medición en la ADM máxima de flexión e extensión de la rodilla, para que se pueda evaluar su flexibilidad. Otra sugerencia se refiere a la verificación de datos de validez y su repetición en medidas de ADM máximas, utilizando otras articulaciones y planos de movimiento. 
COELHO R.R.; BATISTA L.A.; CUNHA G.G. \& DANTAS E.H.M. Validez del uso de la fotografía computarizada en la evaluación de la amplitud del movimiento de la rodilla utilizando la goniometría de la imagen radiológica como padrón oro..Mot. Hum. 13 (1): 45-50; 2012.

\section{REFERENCIAS BIBLIOGRÁFICAS}

1. Berral De La Rosa F, Peroni L, Berral De La Rosa C, Rojano D, Carpintero P. Morfología del miembro inferior y lesiones por sobrecarga. Revista Motricidad Humana 2009: 6(1) 12-8

2. Freitag S, Ellegast R. Dulon M, Nienhaus A. A quantitative measurement of stressful trunk postures in nursing professions. Annals of Occupational Hygiene 2007: 51(4) 385-95.

3. Sprigle AS, Flinn BN, Wootten CM, Mccorry BS. Development and testing of a pelvic goniometer designed to measure pelvic tilt and hip flexion. Clinical Biomechanics 2003: 18(5) 462-5.

4. Coelho RR, Coelho DR, Cunha GG, Dantas EHM. Use of computer aided design system to evaluate the range of motion of the knee in the sagittal plane. Virtual Reality 2011: 4(2) 43-57.

5. Raff GL, Chinnaiyan KM, Share DA, Goraya TY, Kazerooni EA, Moscucci M, Gentry Re, Abidov A. Radiation dose from cardiac computed tomography before and after implementation of radiation dose reduce techniques. JAMA 2009: 301(22) 2340-8.

6. Shiratsu A, Coury H JCG. Reliability and accuracy of different sensors of a flexible electrogoniometer. Clinical Biomechanics 2003: 18(7) 682-4.

7. George K, Batterham A, Sullivan I. Validity in clinical research: a review of basic concepts and definitions. Physical Therapy in Sport 2003 : 4(3), 115-21

8. Humphreys BK, Delahaye M, Peterson CK. An investigation into the validity of cervical spine motion palpation using subjects with congenital block vertebrae as a 'gold standard'. BMC Musculoskeletal Disorders 2004: 19(5), 1-6.

9. Batterham AM, George K. Reliability in evidence-based clinical practice: a primer for allied health professionals. Physical Therapy in Sport 2003: 4(3) 122-8.

10. French SD, Green S, Forbes A. Reliability of chiropractic methods commonly used to detect manipulable lesions in patients with chronic low-back pain. Journal of Manipulative and Physiological Therapeutics 2000: 23(4), 231-8

11. Rosenman MA, Smith G, Maher ML, Ding L, Marchant D. Multidisciplinary collaborative design in virtual environments. Automation in Construction 2007: 16(1) 37-44.
12. Capozzo A, Della Croce U, Leardini A, Chiari L, Human movement analysis using stereophotogrammetry: Part 1: theoretical background. Gait \& Posture 2005: 21(2) 186-96.

13. Seffinger MA, Najm WI, Mishra SI, Adams A, Dickerson Vm, Murphy Ls, Reinsch S. Reliability of spinal palpation for diagnosis of back and neck pain. Spine 2004: 29(19), E413-25.

14. D'apuzzo N. Surface measurement and tracking of human body parts from multi-image video sequences. Journal of Photogrammetry \& Remote Sensing 2002: 56(5-6) 360-75.

15. WORLD MEDICAL ASSOCIATION (WMA). Declaration of Helsinki. Ethical Principles for Medical Research Involving Human Subjects. 59th WMA General Assembly, Seoul, South Korea; 2008.

16. Brosseau L, Balmer S, Tousignant M, P, Goudreault C, Goudreault $\mathrm{M}$, Gringras S. Intra and intertester reliability and criterion validity of the parallelogram and universal goniometers for measuring maximum active knee flexion and extension of patients with knee restrictions. Archives of Physical Medicine Rehabilitation 2001: 82(3), 396-402.

17. Paterniani PES, Barros RML, Brenzikofer R, Lima Filho EC. Metodologia para reconstrução tridimensional e análise da superfície do tronco humano. Brazilian Journal of Biomechanics 2001: 3(1) 51-6.

18. Zhang Z. A Flexible New Technique for Camera Calibration. Microsoft Research, Microsoft Corporation, California USA; 1998.

19. Brodie Da. Mellen Studies in Education Volume 26: A Reference Manual for Human Performance Measurement in the field of Physical Education and Sports Science. The Edwin Mellen Press, New York USA; 1996.

20. Schwartz MH, Rozumalski A. A new method for estimating joint parameters from motion data. Journal of Biomechanics, 2005: 38(1) 107-16.

21. Leardini A, Chiari L, Della Croce U, Cappozzo A. Human movement analysis using stereophotogrammetry. Part 3. Soft tissue artifact assessment and compensation. Gait \& Posture 2005: 21(2) 212-25.

22. Debeliso M, O'shea JP, Harris C, Adams KJ, Climstein M. The relation between trunk strength measures and lumbar disc deformation during stoop type lifting. Journal of Exercise Physiology 2004: 7(6), 16-26.

\section{Dirigir Correspondencia a:}

Renato Coelho

renatorcoelho@coc.ufrj.br

Calle Bernardo Guimarães 1861/403, Lourdes, Belo Horizonte, Minas Gerais, Brasil.

Tel: +553132912395

Móvil: +553199672365

\section{RECIBIDO 8-04-2012}

ACEPTADO: 3-06-2012 